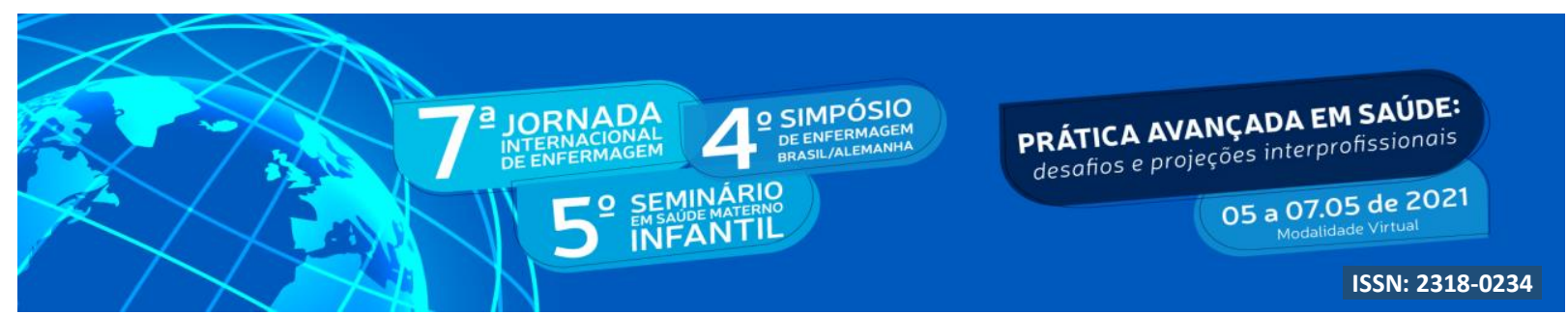

DOI: http://doi.org/10.48195/jie2021-037

\title{
QUALIDADE DO SONO DE JOVENS MILITARES DURANTE PANDEMIA COVID191
}

\author{
Vinícius Iahnke $^{2}$; Cristina Moraes ${ }^{3}$
}

\begin{abstract}
RESUMO
O objetivo deste trabalho foi avaliar a qualidade do sono de jovens militares do Colégio Militar de Santa Maria (CMSM). Para atingir o objetivo, a amostra foi delimitada em militares homens de 18 a 21 anos, servindo no CMSM no ano de 2020 para geração do Índice de qualidade do sono (PSQI). A amostragem é não-probabilística, por conveniência. O tamanho amostral foi 68 participantes. Para avaliação da qualidade do sono, foi utilizado o Pittsburgh Sleep Quality Index (PSQI). Resultados: observou-se que 66,2\% dos militares apresentaram alterações na qualidade do Sono, $61,7 \%$ apresentou qualidade do sono ruim e $4,5 \%$ presença de distúrbios no sono. O sono bom foi observado em $33,8 \%$ dos militares. Conclui-se que a prevalência da qualidade do sono ruim é elevada entre os jovens militares, com o aparecimento de distúrbios do sono em alguns casos. $\mathrm{O}$ isolamento social e o consumo de álcool podem ter influenciado o resultado.
\end{abstract}

Palavras-chave: Sono; Covid-19; Álcool; Pittsburg.

\begin{abstract}
The objective of this work was to evaluate the quality of sleep of young soldiers of the Colégio Militar de Santa Maria (CMSM). To achieve the objective, the sample was delimited in military men aged 18 to 21 years, serving in the CMSM in 2020 to generate the Sleep Quality Index (PSQI). Sampling is non-probabilistic, for convenience. The sample size was 68 participants. To assess sleep quality, the Pittsburgh Sleep Quality Index (PSQI) was used. Results: it was observed that $66.2 \%$ of the military showed changes in sleep quality, $61.7 \%$ had poor sleep quality and $4.5 \%$ had sleep disturbances. Good sleep was observed in $33.8 \%$ of the military. It is concluded that the prevalence of poor sleep quality is high among young soldiers, with the appearance of sleep disorders in some cases. Social isolation and alcohol consumption may have influenced the outcome.
\end{abstract}

Key Words: Sleep; Covid-19; Alcohol; Pittsburg.

\footnotetext{
${ }^{1}$ Trabalho vinculado ao projeto de pesquisa do Mestrado de Ciências da Saúde e da Vida UFN

${ }^{2}$ Estudante do Curso de Mestrado em Ciências da Saúde e da Vida. UFN. E-mail: iahnke13@gmail.com

${ }^{3}$ Orientador. Profa. Dra. Cristina Machado Bragança de Moraes. UFN. E-mail: c_bmoraes@yahoo.com.br
} 


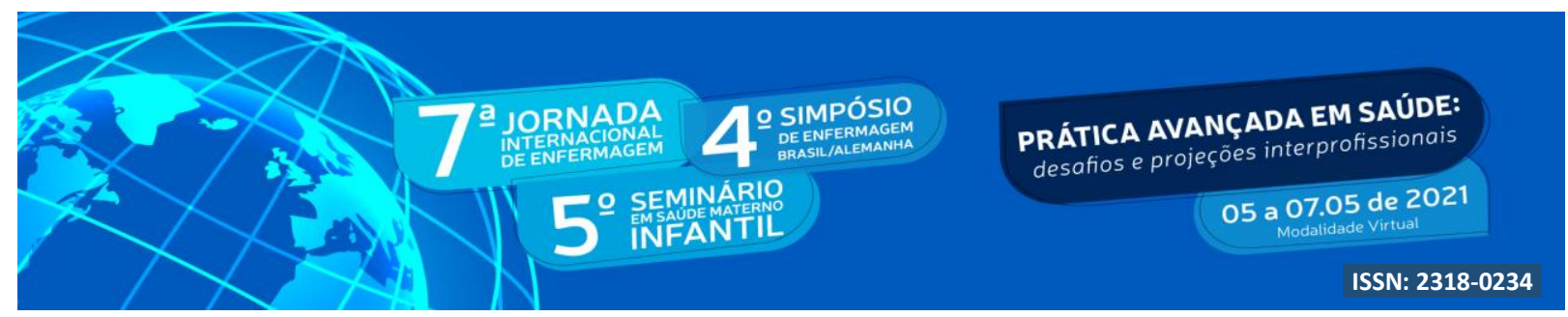

\section{INTRODUÇÃO}

Para o desempenho adequado da profissão militar é fundamental que os integrantes das diversas forças armadas e policiais estejam preparados para enfrentarem possíveis agentes estressores físicos, psicológicos, ambientais ou nutricionais, estando assim preparados para a atuação em condições extremas e que necessitem de versatilidade e adaptabilidade (BRASIL, 2015).

Neste sentido, o sono apresenta-se como um mecanismo fundamental na manutenção da saúde física e mental uma vez que é definido, segundo Carskadon, Dement e Kryger (2005), como um estado neurocomportamental recorrente e reversível, de relativo desengajamento perceptivo de falta de resposta ao meio ambiente, tipicamente acompanhado por decúbito postural, quietude comportamental e olhos fechados; período este, responsável pela restauração do organismo através da produção de hormônios e manutenção da energia corporal.

Apesar do sono ser reconhecido como um mecanismo vital para a saúde física, cognitiva e emocional, a incidência dos transtornos relacionados ao sono vem aumentando substancialmente ao redor do mundo. Nos EUA, estudos apontam que 43 milhões de pessoas sofrem com distúrbios relacionados a perda ou sono entrecortado, e as alterações afetam diretamente a saúde através de reflexos na resistência insulínica, hipertensão, diabetes e obesidade (HIRSHKOWITZ et al., 2015; MCARDLE; KATCH; KATCH, 2015).

A atividade militar possui características peculiares que impõem algumas situações de exposição ao risco e restrições de sono em função das atribuições profissionais. Em 2014, 14\% dos militares do Exército Americano foram diagnosticados com transtornos do sono, apesar de indivíduos com carência crônica de sono possuírem maior probabilidade de sofrer de doenças crônicas não transmissíveis e de desencadearem alterações cognitivas que podem colocar em risco a atividade militar. (SILVA et al., 2014; THOMPSON; JONES; THORNBURG, 2017).

Para o tratamento dos distúrbios relacionados ao sono existem intervenções com 


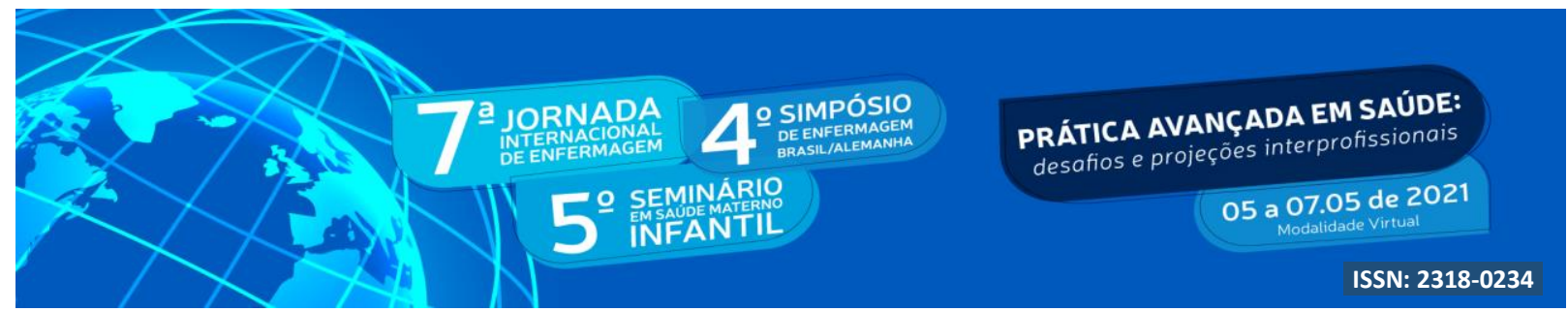

utilização de medicamentos e as não medicamentosas. As que utilizam os fármacos tem objetivo de sedação, provocando redução da ansiedade e relaxamento; dentre as intervenções não medicamentosas, diversas intervenções são apontadas como produtoras de efeitos benéficos, dentre as opções seguras e aparentemente eficazes destacam-se a terapia cognitivocomportamental, terapia de controle de estímulos, terapia de restrição de sono, terapia de relaxamento e o exercício físico (BERLIM; LOBATO; MANFRO, 2005; MCARDLE; KATCH; KATCH, 2015; RIBEIRO, 2016; TYNJALA, 1999).

Fruto da grande relevância da saúde física e mental para a atividade militar, principalmente pelas características atípicas da profissão que exigem uma condição física e mental apropriada, o presente estudo teve por objetivo verificar o índice de qualidade do sono de jovens militares do Colégio Militar de Santa Maria - RS. Com o resultado deste estudo objetivou-se gerar evidências de confiabilidade que servem como instrumento de consulta e auxilio no planejamento das atividades físicas do Exército Brasileiro, no âmbito da atividade física, repouso, preparo físico e mental para a atuação no serviço militar.

\section{OBJETIVO}

Verificar o Índice de qualidade do sono de militares do Colégio Militar de Santa Maria

\section{METODOLOGIA}

Este estudo é do tipo metodológico quantitativo transversal. O presente trabalho foi submetido à apreciação do Comitê de Ética em Pesquisa com Seres Humanos da Universidade Franciscana, e teve início após sua aprovação CAAE: 38712320.9.000.5306 parecer: 4.350.381, assim como, após a assinatura do Termo de Consentimento Livre e Esclarecido por todos os participantes. A pesquisa está enquadrada nas diretrizes e normas regulamentadoras de pesquisas com seres humanos conforme Resolução $n^{\circ} 466$, de 12 de dezembro de 2012 do Conselho Nacional de Saúde.

O estudo desenvolveu-se no Colégio Militar de Santa Maria - RS. Na instituição, anualmente, prestam serviço militar obrigatório aproximadamente 80 militares. Este 


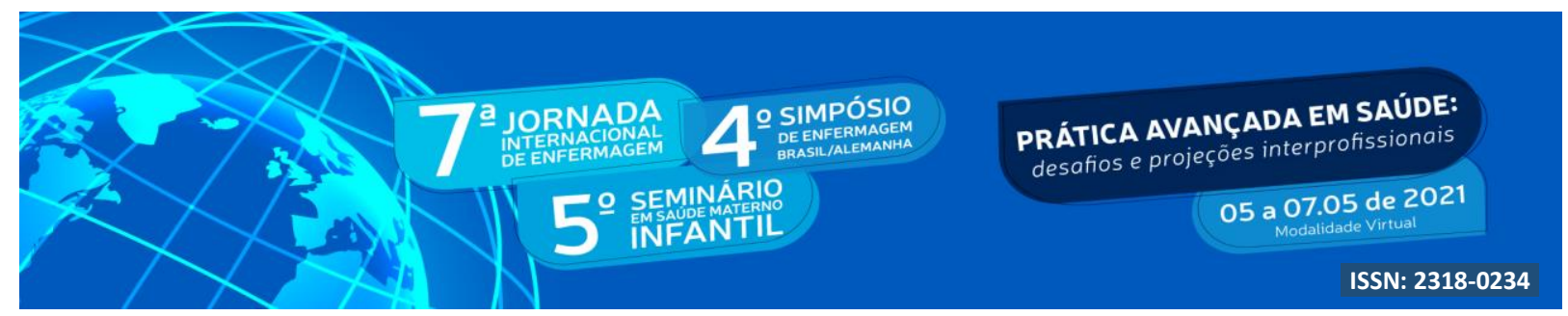

contingente apoia as atividades manutenção, segurança e serviços gerais da unidade.

A amostra foi delimitada por militares homens de 18 a 21 anos, servindo no Colégio Militar de Santa Maria - RS no ano de 2020, na geração dos dados referentes ao Índice de qualidade do sono (PSQI). O efetivo total destes militares é de 80 pessoas. A amostragem é não-probabilística, por conveniência. O cálculo do tamanho amostral foi realizado via software G*Power (FAUL et al., 2007); com os parâmetros: $\alpha=0,05,1-\beta=0,95$. O tamanho amostral mínimo recomendado foi de 67 participantes.

Para avaliação da qualidade do sono, foi utilizado o Pittsburgh Sleep Quality Index (PSQI), questionário possui 19 questões referentes a qualidade e distúrbios de sono no último mês que avaliam sete componentes do sono: 19 qualidade subjetiva, latência do sono, duração do sono, eficiência do sono, distúrbios do sono, uso de medicamentos e disfunção diária. Para cada componente o escore pode variar de 0 a 3, chega-se a um escore de no máximo 21 pontos. O escore do PSQI foi classificado da seguinte forma: $\leq 4$ qualidade do sono boa; $5 \leq$ escore $\leq 10$ qualidade do sono ruim; $11 \leq$ presença de distúrbios do sono (BERTOLAZI et al., 2011; BUYSSE et al., 1989).

Para a coleta dos dados da pesquisa, os militares componentes da amostra foram convidados oralmente a participar do trabalho na reunião matinal. Foram apresentados os objetivos da pesquisa, os procedimentos de coleta realizados, e a contribuição individual e institucional proporcionada pelo estudo. Os participantes foram convidados, de forma voluntária, a dirigirem-se para o Seção de Educação Física do Colégio Militar de Santa Maria - RS, em grupos de até 10 militares, para responderem um questionário de anamnese inicial e o questionário da Qualidade do Sono de Pittsburgh (PSQI-BR). Antes do início do preenchimento do questionário, o pesquisador apresentou o Termo de Consentimento Livre e Esclarecido - TCLE e realizou a leitura de todos os itens dos questionários em voz alta aos participantes, de forma que fossem sanadas possíveis dúvidas antes do início das atividades.

\section{RESULTADOS E DISCUSSÃO}

Foram avaliados 68 militares do efetivo variável prestando serviço militar obrigatório no ano de 2020. A idade média foi de 19,08 \pm 0,6166 anos e todos eram do sexo masculino. 


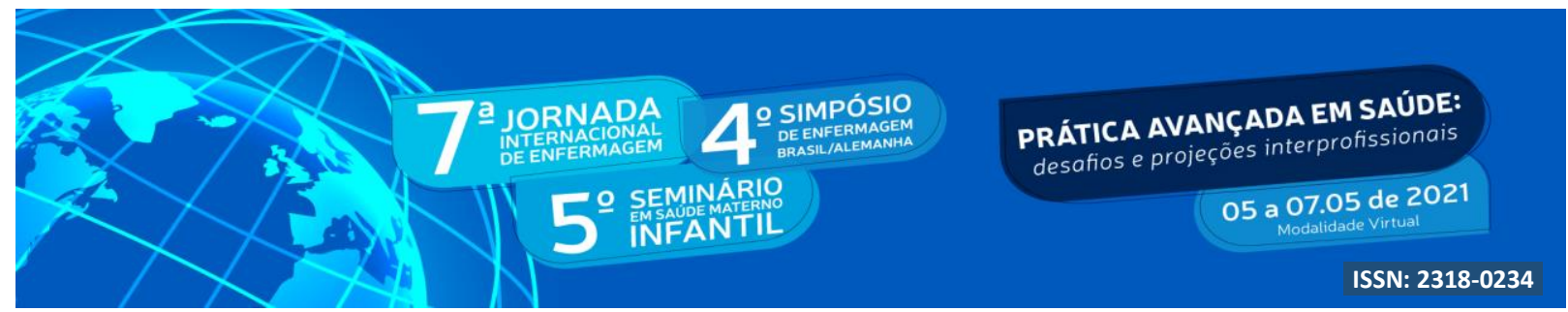

Todos relataram trabalhar em sistema de escala de serviço com plantões de 24 horas pelo menos uma vez por semana.

A partir da análise do questionário da Qualidade do Sono de Pittsburgh (PSQI-BR) e da classificação do sono da amostra, observou-se que 33,8\% dos militares que compõem a amostra apresentam qualidade do sono boa, $61,7 \%$ apresentaram qualidade do sono ruim e 4,5\% dos militares apresentaram presença de distúrbios do sono.

Possíveis fatores intervenientes na qualidade do sono foram avaliados na anamnese inicial, de forma que $91,1 \%$ da amostra foi composta por não fumantes e $8,9 \%$ de fumantes. No quesito consumo de álcool, 35,3\% da amostra não consome bebidas alcoólicas, 55,8\% apresenta comportamento de baixo consumo pois ingere bebidas alcoólicas de 1-2 vezes por semana, 7,3\% da amostra apresenta comportamento de consumo alcoólico médio, de 3-4 vezes por semana e 1,6\% apresenta comportamento de alto consumo alcoólico, consumindo 5 ou mais vezes por semana.

Analisando os resultados, observa-se que uma grande parcela dos jovens militares possui problemas com o sono, sendo que $66,2 \%$ da amostra foi classificada com qualidade sono ruim ou identificada com presença de distúrbios do sono. O resultado vai ao encontro dos resultados de estudos com base populacional que apontam para índices superiores a $46,7 \%$ da população brasileira com algum tipo de problema com o sono. Nos EUA, os problemas relacionados ao sono quebrado atingem 43 e 20 milhões de pessoas, respectivamente (MCARDLE; KATCH; KATCH, 2015; PINTO et al., 2018).

Apesar da grande incidência de problemas relacionados ao sono, o período necessário de sono para restauração é de sete a nove horas para jovens adultos entre 18 e 25 anos, porém este período não é normalmente atingido pelos grupos populacionais. No meio militar, as alterações no sono podem colorar em risco a execução de tarefas, expondo os integrantes, por vezes, ao risco de vida; uma vez que as alterações relacionadas a qualidade do sono podem ocasionar redução no desempenho cognitivo, redução na necessária atenção durante as tarefas e interferir negativamente na capacidade de resolver problemas (HIRSHKOWITZ et al., 2015; OHAYON et al., 2017; TROXEL et al., 2015; WOLFSON et al., 2007).

Em relação ao consumo de álcool, apesar de estudos apontarem para maiores índices de consumo alcoólico no fim da adolescência, foi possível observar um comportamento de 


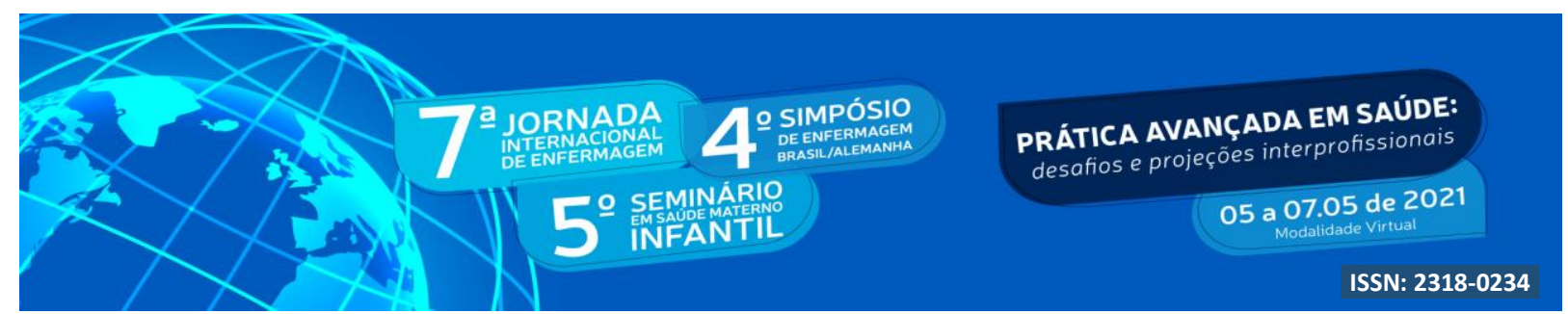

consumo superior quando comparamos com dados de estudos populacionais recentes para a mesma faixa etária no Brasil. Em 2019, segundo Pesquisa Nacional de Saúde realizada pelo IBGE, a proporção de homens que possuem o hábito de consumir bebida alcoólica ao menos uma vez por semana é de $37,1 \%$, comparando com resultado da amostra estudada, verifica-se que o número de jovens militares que consomem bebidas alcoólicas ao menos 1 vez por semana é de $64,7 \%$, representando um número expressivamente maior de consumidores de álcool (ABREU et al., 2020; MINISTÉRIO DA SAÚDE, 2020).

Deve-se levar em consideração para a discussão dos resultados do estudo que o período da coleta de dados da pesquisa foi entre dezembro/2020 e janeiro/2021; momento ímpar na história da saúde pública mundial que requer uma discussão e análise cuidadosa dos dados pois o Brasil atravessa uma grave crise de saúde pública decorrente da pandemia COVID19. Medidas restritivas de circulação, incentivo ao isolamento social, trabalho remoto e cancelamento de eventos presenciais em grande parte das cidades são características do período e que podem influenciar diretamente as variáveis estudadas pois afetam o convívio social e a resposta indivídual através de reflexos no sono e no comportamento.

\section{CONCLUSÃO}

As evidências encontradas a partir deste estudo mostram que a prevalência da qualidade do sono ruim é elevada entre os jovens militares, inclusive com o aparecimento de distúrbios do sono em alguns casos. O consumo de álcool pelos integrantes da amostra foi superior ao de jovens avaliados em estudos populacionais com a mesma faixa etária. $\mathrm{O}$ período de isolamento social provocado pela pandemia da COVID19 no momento da coleta dos dados da pesquisa pode ter influenciado no comportamento de consumo de álcool, cigarros e na qualidade do sono da amostra. A relação entre a prevalência da má qualidade do sono e o consumo de álcool pode ser explorada em estudos futuros com a amostra, bem como a influência do período de pandemia e reclusão social nas variáveis do estudo. 


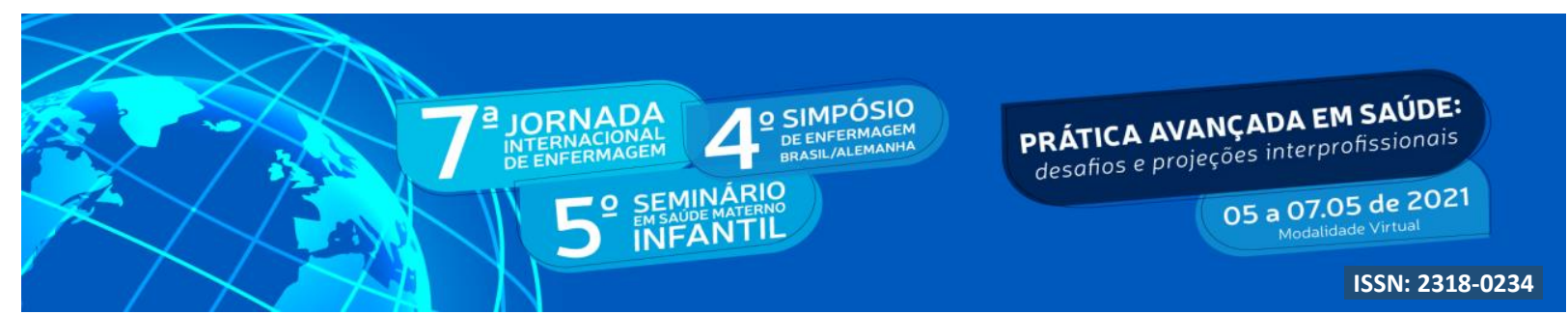

\section{REFERÊNCIAS}

ABREU, M. N. S. et al. Prevalência e fatores associados ao consumo excessivo episódico de álcool entre adultos jovens brasileiros de 18 a 24 anos. Revista Brasileira de Epidemiologia, v. 23, p. e200092, 2020.

BERLIM, M. T.; LOBATO, M. I.; MANFRO, G. Diretrizes e algoritmo para o manejo da insônia. In: Psicofármacos: Consulta Rápida. Porto Alegre: Artmed, 2005.

BERTOLAZI, A. N. et al. Validation of the Brazilian Portuguese version of the Pittsburgh Sleep Quality Index. Sleep Medicine, v. 12, n. 1, p. 70-75, 2011.

BRASIL. MINISTÉRIO DA DEFESA. EXÉRCITO BRASILEIRO. ESTADO MAIOR DO EXÉRCITO. Manual de Campanha EB20-MC-10.350 - Treinamento Físico Militar, 2015.

BUYSSE, D. J. et al. The Pittsburgh sleep quality index: A new instrument for psychiatric practice and research. Psychiatry Research, v. 28, n. 2, p. 193-213, 1989.

CARSKADON, M. A.; DEMENT, W. C.; KRYGER, M. Principles and Practice of Sleep Medicine. 4. ed. Philadelphia: Elsevier Saunders, 2005.

HIRSHKOWITZ, M. et al. National Sleep Foundation's sleep time duration recommendations: methodology and results summary. Sleep Health, v. 1, p. 40-43, 2015.

MCARDLE, W.; KATCH, F.; KATCH, V. Exercise Phisiology: Nutrition, Energy, and Human Performance. 8. ed. Philadelphia: Wolters Kluwer Health/Lippincott Williams \& Wilkins, 2015.

MINISTÉRIO DA SAÚDE. Pesquisa Nacional de Saúde 2019, 2020.

OHAYON, M. et al. National Sleep Foundation's sleep quality recommendations: first report. Sleep Health, v. 3, n. 1, p. 6-19, 2017.

PINTO, J. DO N. et al. Avaliação do Sono em um Grupo de Policiais Militares de Elite. Acta Paulista de Enfermagem, v. 31, n. 2, p. 153-161, mar. 2018.

RIBEIRO, N. F. Tratamento da Insônia em Atenção Primária à Saúde. Revista Brasileira de Medicina de Família e Comunidade, v. 11, n. 38, p. 1-14, 2016.

SILVA, L. R. DA et al. Fatores de risco para hipertensão arterial em policiais militares do centro-sul piauiense. Revista Baiana Saúde Pública, v. 38, n. 3, p. 679-692, 2014.

THOMPSON, M. A.; JONES, C. B.; THORNBURG, C. J. Aperfeiçoando a Gestão de Combatentes. MILITARY REVIEW, p. 8, 


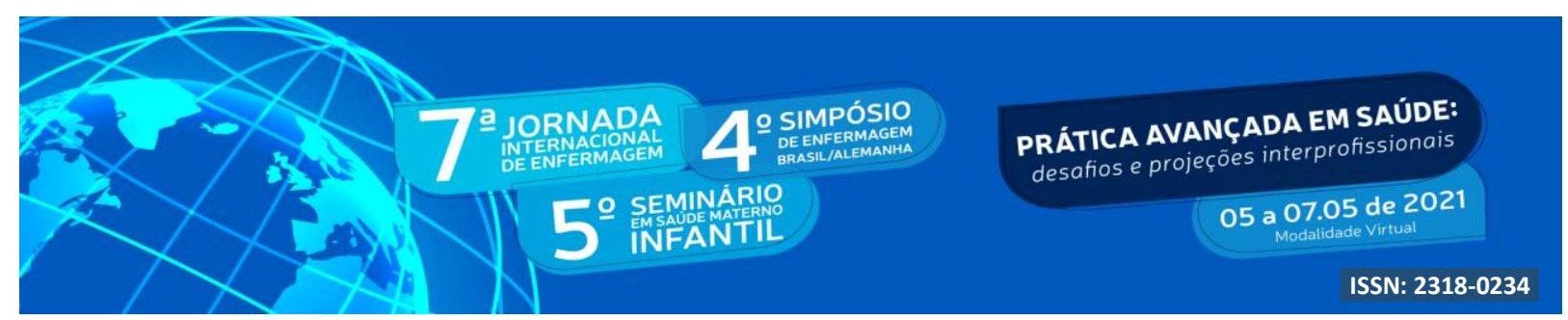

2017.

TROXEL, W. M. et al. Sleep in the Military: Promoting Healthy Sleep Among U.S. Servicemembers. Rand health quarterly, v. 5, n. 19, 2015.

TYNJALA, J. Perceived sleep quality and its precursors in adolescents. Health Promotion International, v. 14, n. 2, p. 155-166, 1 jun. 1999.

WOLFSON, A. R. et al. Middle School Start Times: The Importance of a Good Night's Sleep for Young Adolescents. Behavioral Sleep Medicine, v. 5, n. 3, p. 194-209, 2007. 